\title{
Optimizing fluorescence guidance in sentinel node biopsy
}

Sentinel node biopsy aims to minimize the need for extensive pelvic node dissection by identifying those nodes that are most likely to contain metastases. Currently, the identification of the sentinel node using fluorescence imaging is hindered by poor penetration of the commonly used indocyanine green (ICG) dye, which prevents preoperative mapping and means that mapping of the entire pelvic region must be carried out during surgery. New research published in European Urology describes the stepwise optimization of a hybrid tracer approach using ICG- ${ }^{99 \mathrm{~m}} \mathrm{Tc}-$ nanocolloid to integrate fluorescence guidance and preoperative imaging.

The study included 40 patients with localized prostate cancer and $>10 \%$ risk of metastases as estimated using the Briganti nomogram. Patients were split into three treatment groups. Group 1 received a previously described hybrid tracer, whereas groups 2 and 3 used a new tracer formulation of ICG- ${ }^{-99 \mathrm{~m}} \mathrm{Tc}$-nanocolloid. In Group 2, the new tracer was used

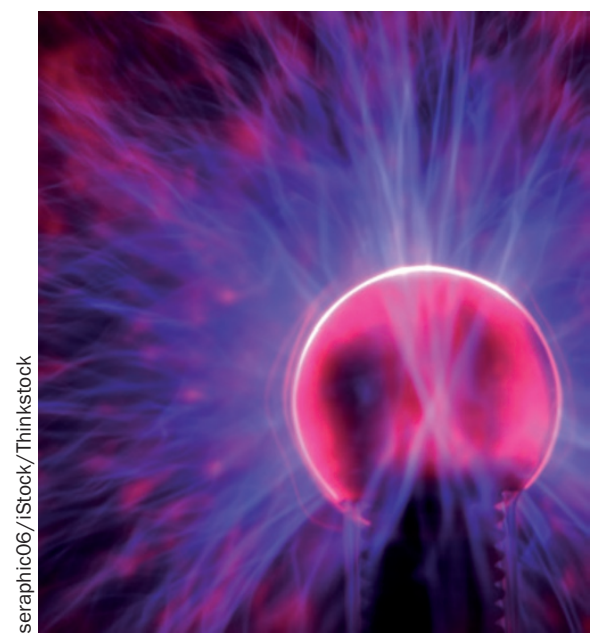

using static planar lymphoscintigraphy $15 \mathrm{~min}$ and $2 \mathrm{~h}$ after injection, followed by SPECT and low-dose CT scan.

At least one sentinel node was identified in 38 of the 40 patients using preoperative imaging. Mean fluorescencebased sentinel node identification increased overall from $63.7 \%$ in Group 1 to $85.2 \%$ and $93.5 \%$ in Groups 2 and 3, respectively. Postoperative complications did not differ between groups, but Kaplan-Meier analysis showed an improvement in biochemical-recurrence (BCR)-free-survival in Group 3.

The authors assert that this stepwise optimization of the flourescence-guided technique could represent better nodal staging and, potentially, improve BCR-free survival in patients with prostate cancer. and reduced injection volume, and in Group 3, an upgraded laparoscopic fluorescence-imaging system was added. Tracer was injected into the peripheral zone of the prostate transrectally under ultrasonographic guidance. Preoperative sentinel node mapping was carried out

\section{Annette Fenner}

Original article KleinJan, G. H. et al. Optimisation of fluorescence guidance during robot-assisted laparoscopic sentinel node biopsy for prostate cancer. Eur. Urol. doi:10.1016/j.eururo.2014.07.014 